\title{
Reduction and construction of Poisson quasi-Nijenhuis manifolds with background
}

\author{
Flávio Cordeiro $†$ and Joana M. Nunes da Costa $\ddagger$ \\ $\dagger$ Mathematical Institute, University of Oxford, England \\ $\ddagger$ CMUC, University of Coimbra, Portugal
}

October 25, 2018

\begin{abstract}
We extend the Falceto-Zambon version of Marsden-Ratiu Poisson reduction to Poisson quasi-Nijenhuis structures with background on manifolds. We define gauge transformations of Poisson quasi-Nijenhuis structures with background, study some of their properties and show that they are compatible with reduction procedure. We use gauge transformations to construct Poisson quasi-Nijenhuis structures with background.
\end{abstract}

\section{Introduction}

Poisson quasi-Nijenhuis structures with background were recently introduced by Antunes [1] and include, as a particular case, the Poisson quasiNijenhuis structures defined by Stiénon and Xu [15]. The structure consists of a Poisson bivector together with a $(1,1)$-tensor and two closed 3-forms fulfilling some compatibility conditions. In [21], Zucchini showed that some physical models provide a structure which is a bit more general than Poisson quasi-Nijenhuis manifolds with background. In fact, as it is observed in [1, comparing with our definition, in Zucchini's definition one condition is missing. Generalized complex structures with background, also called twisted generalized complex structures, are another special case of Poisson quasiNijenhuis structures with background. They were introduced by Gualtieri [7] and further studied, among other authors, by Lindström et al [11] and Zucchini [21] in relation with sigma models in physics.

In order to simplify the writing, we will use $\mathrm{PqNb}$ for Poisson quasiNijenhuis with background, $\mathrm{PqN}$ for Poisson quasi-Nijenhuis, PN for PoissonNijenhuis and gc for generalized complex. 
The aim of this paper is two fold. Firstly, we study reduction of $\mathrm{PqNb}$ manifolds and secondly, by means of a technique that we call gauge transformation, we are able to construct these structures from simpler ones. Moreover, we prove that these two procedures are compatible in the sense that they commute.

One of our goals is to extend Poisson reduction to PqNb structures. The classical Marsden-Ratiu [13] method of Poisson reduction by distributions was recently reformulated by Falceto and Zambon [6] and it is this new version of Poisson reduction that we apply to $\mathrm{PqNb}$ structures. Our scheme is the following: reduce the Poisson bivector on the manifold and then establish the conditions ensuring that the remaining tensor fields that define the $\mathrm{PqNb}$ structure also descend to the quotient in such a way that the reduced structure is in fact a $\mathrm{PqNb}$ structure.

In this paper we view gc structures with background as particular cases of $\mathrm{PqNb}$ structures. Thus, in a very natural way, gc structures with background gain a reduction procedure which turns to be a generalization of Vaisman's reduction theorem of gc structures (Theorem 2.1 in [19]). There are other different approaches to reduction of gc structures and gc structures with background (see [3, 9, 10, 16, 20]).

Besides reduction, the other main notion in this paper is gauge transformation. Inspired by the corresponding notion for gc structures, also called $B$-field transformation, we define gauge transformations of $\mathrm{PqNb}$ structures and realize that they can be seen as a tool for constructing PqNb structures from other $\mathrm{PqNb}$ structures. In particular, we may construct richer examples of such structures from simpler ones and, indeed, we construct a new class of $\mathrm{PqNb}$ structures by applying gauge transformations to the simplest $\mathrm{PqNb}$ structures, i.e. those consisting just of a Poisson bivector. Unlike gauge transformations of Dirac structures which are graphs of Poisson bivectors 14, 2, our notion of gauge transformation preserves the Poisson bivector of the $\mathrm{PqNb}$ structure. Moreover, these gauge transformations share very interesting properties, some of them we discuss, and which may be used to study the class of all $\mathrm{PqNb}$ structures on a given manifold. We should mention that, in [21], Zucchini gives a similar definition of gauge transformation with respect to the structure defined there, but he doesn't present the proof that the gauge transformations preserve such structure.

The paper is organized as follows. In section $1, \mathrm{PqNb}$ structures and gc structures with background are recalled. Section 2 is devoted to reduction. After a brief review of Poisson reduction, in the sense of Falceto-Zambon, we give a reduction theorem for $\mathrm{PqNb}$ manifolds and we also discuss the case of reduction by a group action. Still in section 2, we treat the reduction of gc structures with background. In section 3, we introduce the concept of gauge transformation of $\mathrm{PqNb}$ structures and we show how to use it to construct richer examples of $\mathrm{PqNb}$ structures from simpler ones. We also consider conformal change by Casimir functions and, combining it with 
gauge transformation, we obtain new examples of $\mathrm{PqNb}$ structures. We study some properties of gauge transformations and, finally, we show that gauge transformations commute with reduction. The paper closes with an appendix containing the proof of some technical lemmas.

\section{Preliminaries}

\subsection{Poisson quasi-Nijenhuis manifolds with background}

Let us recall that a bivector field $Q \in \mathfrak{X}^{2}(M)$ on a $C^{\infty}$-differentiable manifold $M$ determines a bracket [, $]_{Q}$ of 1 -forms $\alpha, \beta \in \Omega^{1}(M)$ :

$$
[\alpha, \beta]_{Q}=\mathcal{L}_{Q^{\sharp} \alpha}(\beta)-\mathcal{L}_{Q^{\sharp} \beta}(\alpha)-d(Q(\alpha, \beta)) .
$$

Given a (1,1)-tensor $A$ on $M, A: T M \rightarrow T M,[,]_{A}$ denotes the bracket of vector fields $X, Y \in \mathfrak{X}^{1}(M)$ deformed by $A$ :

$$
[X, Y]_{A}=[A X, Y]+[X, A Y]-A[X, Y],
$$

$\imath_{A}$ is the 0 -degree derivation on the graded algebra $(\Omega(M), \wedge)$ given by

$$
\begin{aligned}
& \left(\imath_{A} \alpha\right)\left(X_{1}, X_{2}, \ldots, X_{k}\right)=\alpha\left(A X_{1}, X_{2}, \ldots, X_{k}\right) \\
& +\alpha\left(X_{1}, A X_{2}, \ldots, X_{k}\right)+\cdots+\alpha\left(X_{1}, X_{2}, \ldots, A X_{k}\right)
\end{aligned}
$$

and $d_{A}$ is the derivation of degree 1 on $(\Omega(M), \wedge)$ given by

$$
d_{A}=\left[\imath_{A}, d\right]=\imath_{A} \circ d-d \circ \imath_{A} .
$$

The Nijenhuis torsion of $A$ is the (1,2)-tensor $\mathcal{N}_{A}$ defined by

$\mathcal{N}_{A}(X, Y)=[A X, A Y]-A([A X, Y]+[X, A Y]-A[X, Y]), X, Y \in \mathfrak{X}^{1}(M)$.

For the bracket $[,]_{A}$ on $\mathfrak{X}^{1}(M)$, the corresponding bracket of 1-forms determined by the bivector $Q$, which we denote by $\left([,]^{A}\right)_{Q}$, is given by (1) where $d$ is replaced by $d_{A}$ and $\mathcal{L}$ by $\mathcal{L}^{A}, \mathcal{L}_{X}^{A}=\imath_{X} \circ d_{A}+d_{A} \circ \imath_{X}$. The concomitant of $P$ and $A$ is the $(2,1)$-tensor $\mathcal{C}_{P, A}$ given by

$$
\mathcal{C}_{P, A}(\alpha, \beta)=\frac{1}{2}\left(\left([\alpha, \beta]^{A}\right)_{P}-\left([\alpha, \beta]_{P}\right)_{A^{t}}\right),
$$

where $A^{t}: T^{*} M \rightarrow T^{*} M$ denotes the transpose of $A$ and $\left([,]_{P}\right)_{A^{t}}$ is the bracket $[,]_{P}$ deformed by $A^{t}$. This is equivalent to

$$
\begin{aligned}
\mathcal{C}_{P, A}(\alpha, \beta)= & \mathcal{L}_{P^{\sharp} \beta}\left(A^{t} \alpha\right)-\mathcal{L}_{P^{\sharp} \alpha}\left(A^{t} \beta\right)+A^{t} \mathcal{L}_{P^{\sharp} \alpha}(\beta)-A^{t} \mathcal{L}_{P^{\sharp} \beta}(\alpha) \\
& +d\left(P\left(A^{t} \alpha, \beta\right)\right)-A^{t} d(P(\alpha, \beta)),
\end{aligned}
$$

for all $\alpha, \beta \in \Omega^{1}(M)$. This concomitant is one half of that defined in [1].

Poisson quasi-Nijenhuis structures with background were recently defined by Antunes in [1]. We now propose a slightly different definition. For the interior product a form $\omega \in \Omega(M)$ by the bivector $X \wedge Y$, we use the convention $\imath_{X \wedge Y} \omega=\imath_{Y} \imath_{X} \omega$. 
Definition 1.1. A Poisson quasi-Nijenhuis structure with background on a manifold $M$ is a quadruple $(P, A, \phi, H)$ of tensors on $M$ where $P$ is a Poisson bivector, $A: T M \rightarrow T M$ is a $(1,1)$-tensor and $\phi$ and $H$ are closed 3-forms, such that

$$
\begin{aligned}
& A \circ P^{\sharp}=P^{\sharp} \circ A^{t}, \\
& \mathcal{C}_{P, A}(\alpha, \beta)=-\imath_{P^{\sharp} \alpha \wedge P^{\sharp} \beta} H, \\
& \mathcal{N}_{A}(X, Y)=P^{\sharp}\left(\imath_{X \wedge Y} \phi+\imath_{A X \wedge Y} H+\imath_{X \wedge A Y} H\right), \\
& d_{A} \phi=d \mathcal{H},
\end{aligned}
$$

for all $X, Y \in \mathfrak{X}^{1}(M), \alpha, \beta \in \Omega^{1}(M)$, and where $\mathcal{H}$ is the 3 -form given by

$$
\mathcal{H}(X, Y, Z)=\circlearrowleft_{X, Y, Z} H(A X, A Y, Z),
$$

for all $X, Y, Z \in \mathfrak{X}^{1}(M)$, the symbol $\circlearrowleft_{X, Y, Z}$ meaning a sum over the cyclic permutations of $(X, Y, Z)$. The 3 -form $H$ is called the background and the manifold $M$ with such structure is said to be a Poisson quasi-Nijenhuis manifold with background.

The difference between this definition and that given in [1] is the minus sign in equation (5). With this change, the definition above contains the class of generalized complex manifolds with background and, moreover, enables us to define the concept of gauge transformations of $\mathrm{PqNb}$ structures.

When $H=0$, this reduces to the Poisson quasi-Nijenhuis structures defined in [15]. If, in addition, $\phi=0$, we get the Poisson-Nijenhuis structures introduced in [12].

A very simple example of a $\mathrm{PqNb}$ structure is the following.

Example 1.2. Consider $\mathbb{R}^{3}$ with coordinates $\left(x_{1}, x_{2}, x_{3}\right)$ and take any $C^{\infty}$ functions $f: \mathbb{R}^{3} \rightarrow \mathbb{R} \backslash\{0\}$ and $g: \mathbb{R}^{3} \rightarrow \mathbb{R}$ such that $\frac{\partial g}{\partial x_{1}}=\frac{\partial g}{\partial x_{2}}=0$ at any point. Then, the quadruple $(P, A, \phi, H)$ with $P=f \frac{\partial}{\partial x_{1}} \wedge \frac{\partial}{\partial x_{2}}$, $A=g\left(\frac{\partial}{\partial x_{1}} \otimes d x_{1}+\frac{\partial}{\partial x_{2}} \otimes d x_{2}+\frac{\partial}{\partial x_{3}} \otimes d x_{3}\right), H=-\frac{1}{f} \frac{\partial g}{\partial x_{3}} d x_{1} \wedge d x_{2} \wedge d x_{3}$ and $\phi=-2 g H$ is a PqNb structure on $\mathbb{R}^{3}$. Notice that $\mathcal{C}_{P, A}(\alpha, \beta)=$ $\frac{\partial g}{\partial x_{3}} P(\alpha, \beta) d x_{3}=-\imath_{P^{\sharp} \alpha \wedge P^{\sharp} \beta} H$, for all $\alpha, \beta \in \Omega^{1}\left(\mathbb{R}^{3}\right)$, and $A$ is a Nijenhuis tensor, $\mathcal{N}_{A}(X, Y)=P^{\sharp}\left(\imath_{X \wedge Y}(\phi+2 g H)\right)=0$, for all $X, Y \in \mathfrak{X}^{1}\left(\mathbb{R}^{3}\right)$.

Remark 1.3. In [21], Zucchini has shown that the geometry of the Hitchin sigma model incorporates all the defining conditions of a Poisson quasiNijenhuis manifold with background except the last one, condition (7). This was what he called an $H$-twisted Poisson quasi-Nijenhuis manifold, which is slightly more general than a Poisson quasi-Nijenhuis manifold with background but does not satisfy some integrability conditions. 
The concept of $\mathrm{PqNb}$ structure on a manifold, given in Definition 1.1, can be generalized for generic Lie algebroids. This was in fact the approach followed in [1] (see also [4]). However, in the case of the results presented here, the generalization is always straightforward so that we prefer to work with the standard Lie algebroid all the time.

\subsection{Generalized complex structures with background}

Let $M$ be a manifold and consider the generalized tangent bundle $\mathbb{T} M=$ $T M \oplus T^{*} M$. This vector bundle is the ambient framework of generalized complex geometry. This is a recent subject introduced by Hitchin [8], and further studied by Gualtieri [7] and other authors, e.g. [5, 11, 16, 19], which contains the symplectic and complex geometries as extreme cases. The Lie bracket of vector fields on $M$ extends to the well-known Courant bracket [ , ] on $\Gamma(\mathbb{T} M)$ :

$$
[X+\alpha, Y+\beta]=[X, Y]+\mathcal{L}_{X} \beta-\mathcal{L}_{Y} \alpha+\frac{1}{2} d(\alpha(Y)-\beta(X)),
$$

for all $X, Y \in \mathfrak{X}^{1}(M)$ and $\alpha, \beta \in \Omega^{1}(M)$. Given a closed 3-form $H$ on $M$, the Courant bracket can be deformed into the Courant bracket with background $H,[,]_{H}$, by simply adding an $H$-dependent term:

$$
[X+\alpha, Y+\beta]_{H}=[X+\alpha, Y+\beta]-\imath_{X \wedge Y} H .
$$

By the well-known Newlander-Nirenberg theorem, a complex structure on $M$ is equivalent to a vector bundle map $J: T M \rightarrow T M$ satisfying $J^{2}=-$ Id and having a null Nijenhuis torsion. The passage to the generalized case is done by substituting the tangent bundle $T M$ by $\mathbb{T} M$ and the bracket of vector fields by the Courant bracket:

Definition 1.4. Given a closed 3-form $H$ on $M$, a generalized complex structure with background $H$ on $M$ is a vector bundle map $\mathcal{J}: \mathbb{T} M \rightarrow \mathbb{T} M$ satisfying $\mathcal{J}^{2}=-\mathrm{Id}$ and such that the following integrability condition holds:

$$
[\mathcal{J} \mu, \mathcal{J} \nu]_{H}-\mathcal{J}[\mathcal{J} \mu, \nu]_{H}-\mathcal{J}[\mu, \mathcal{J} \nu]_{H}-[\mu, \nu]_{H}=0,
$$

for all $\mu, \nu \in \Gamma(\mathbb{T} M)$. The triple $(M, \mathcal{J}, H)$ is called a generalized complex manifold with background $H$ (or an $H$-twisted generalized complex manifold). When $H=0, \mathcal{J}$ is said to be a generalized complex structure and $(M, \mathcal{J})$ a generalized complex manifold.

Remark 1.5. Equivalently, a gc structure with background $H$ on $M$ can be defined as an isotropic complex vector subbundle $L$ of the complexification $\mathbb{T}_{\mathbb{C}} M=\mathbb{T} M \otimes \mathbb{C}$ such that $\mathbb{T}_{\mathbb{C}} M=L \oplus \bar{L}$, where $\bar{L}$ is the conjugate of $L$, and $L$ is involutive with respect to the Courant bracket $[,]_{H}$. The complex Dirac structures $L$ and $\bar{L}$ are the $(+i)$ and $(-i)$-eigenbundles of $\mathcal{J}$. We prefer to use Definition 1.4 since it is the one that provides the natural link with $\mathrm{PqNb}$ structures. 
The following result completely characterizes generalized complex structures with background in terms of classical tensors. It was referred in [11, 19] and it is a simple extension of the analogous result of Crainic [5] for the case $H=0$.

Theorem 1.6. A vector bundle map $\mathcal{J}: \mathbb{T} M \rightarrow \mathbb{T} M$ is a generalized complex structure with background $H$ on $M$ if and only if it can be written in the form

$$
\mathcal{J}=\left(\begin{array}{cc}
A & P^{\sharp} \\
\sigma^{b} & -A^{t}
\end{array}\right),
$$

where $P$ is a bivector on $M, \sigma$ a 2-form on $M$, and $A: T M \rightarrow T M a$ $(1,1)$-tensor, such that:

(1) $P$ is a Poisson bivector;

(2) $A \circ P^{\sharp}=P^{\sharp} \circ A^{t} \quad$ and $\quad \mathcal{C}_{P, A}(\alpha, \beta)=-\imath_{P^{\sharp} \alpha \wedge P^{\sharp} \beta} H$;

(3) $\mathcal{N}_{A}(X, Y)=P^{\sharp}\left(\imath_{X \wedge Y} d \sigma+\imath_{A X \wedge Y} H+\imath_{X \wedge A Y} H\right)$;

(4) the $(0,2)$-tensor $\sigma_{A}$ defined by $\sigma_{A}(X, Y)=\sigma(A X, Y)$ is antisymmetric and satisfies the relation

$$
d \sigma_{A}+H-\imath_{A} d \sigma-\mathcal{H}=0,
$$

where $\mathcal{H}$ is given by (8);

(5) $A^{2}=-\mathrm{Id}-P^{\sharp} \circ \sigma^{b}$.

By comparing this theorem with Definition 1.1, we immediately see that a gc structure with background is a special case of a PqNb structure. Notice that equation (7), with $\phi=d \sigma$, follows from (12). Thus, we can write Theorem 1.6 as follows:

Theorem 1.7. Let $\mathcal{J}: \mathbb{T} M \rightarrow \mathbb{T} M$ be a vector bundle map of the form (11), $\mathcal{J}:=(A, P, \sigma)$. Then, $\mathcal{J}$ is a generalized complex structure with background $H$ on $M$ if and only if $(P, A, d \sigma, H)$ is a Poisson quasi-Nijenhuis structure with background on $M$ and properties 4 and 5 of Theorem 1.6 are satisfied.

Now, let $(P, A, d \sigma, H)$ be a $\mathrm{PqNb}$ structure on $M$ and take a closed 2form $\omega$ on $M$. It is obvious that the replacement of $\sigma$ by $\sigma+\omega$ makes no change in the $\mathrm{PqNb}$ structure. However, if, additionally, conditions 4 and 5 of Theorem 1.6 are satisfied, i.e. $\mathcal{J}_{\sigma}:=(A, P, \sigma)$ is a gc structure with background $H$, one can ask under what conditions is $\mathcal{J}_{\sigma+\omega}:=(A, P, \sigma+\omega)$ still a gc structure with background $H$ on $M$. An immediate computation shows that this happens if and only if the $(0,2)$-tensor $\omega_{A}$ is antisymmetric, $d \omega_{A}=0$ and $P^{\sharp} \circ \omega^{b}=0$. This means that a $\mathrm{PqNb}$ structure on $M$ has more than one gc structure with background associated with it. Also, this defines an equivalence relation on the subclass of all gc structures having the background $H$ and the Poisson bivector $P$. 


\section{Reduction of Poisson quasi-Nijenhuis manifolds with background}

\subsection{Reduction of Poisson manifolds}

A well-known reduction procedure for Poisson manifolds is the one due to Marsden and Ratiu [13. Roughly speaking, given a Poisson manifold $(M, P)$, a submanifold $N$ of $M$ and a vector subbundle $E$ of $\left.T M\right|_{N}$, MarsdenRatiu reduction theorem establishes necessary and sufficient conditions to have a Poisson structure on the quotient $N /(E \cap T N)$. Recently, Falceto and Zambon [6] showed that the assumptions of Marsden-Ratiu theorem are too strong and they gave more flexible hypothesis on the subbundle $E$ still providing a Poisson structure on the quotient submanifold.

Definition 2.1. Let $(M, P)$ be a Poisson manifold, $i_{N}: N \subset M$ a submanifold of $M$ and $E$ a vector subbundle of $\left.T M\right|_{N}$ such that $E \cap T N$ is an integrable subbundle of $T N$ and the foliation of $N$ defined by such subbundle is simple, i.e. the set $Q$ of leaves is a manifold and the canonical projection $\pi: N \rightarrow Q$ is a submersion. The quadruple $(M, P, N, E)$ is said to be Poisson reducible if $Q$ inherits a Poisson structure $P^{\prime}$ defined by

$$
\{f, h\}_{P^{\prime}} \circ \pi=\{F, H\}_{P} \circ i_{N}
$$

for any $f, h \in C^{\infty}(Q)$ and any extensions $F, H \in C^{\infty}(M)$ of $f \circ \pi, h \circ \pi$, respectively, with $d F$ and $d H$ vanishing on $E$.

Using the notation of [6], we denote by $C^{\infty}(M)_{E}$ the following subset of $C^{\infty}(M)$

$$
C^{\infty}(M)_{E}:=\left\{f \in C^{\infty}(M) \mid d f_{\mid E}=0\right\} .
$$

Theorem $2.2([6])$. Let $(M, P)$ be a Poisson manifold, $i_{N}: N \subset M$ a submanifold of $M$ and $E$ a subbundle of $\left.T M\right|_{N}$ as in Definition 2.1. Let $D$ be a subbundle of $\left.T M\right|_{N}$ such that $E \cap T N \subset D \subset E$ and $\mathcal{E} \subset C^{\infty}(M)_{E}$ a multiplicative subalgebra such that the restriction map $i_{N}^{*}: \mathcal{E} \rightarrow C^{\infty}(N)_{E \cap T N}$ is surjective. If $\{\mathcal{E}, \mathcal{E}\} \subset C^{\infty}(M)_{D}$ and $P^{\sharp}\left(E^{0}\right) \subset T N+D$, then $(M, P, N, E)$ is Poisson reducible.

A special case of the theorem above, which is considered in [6], occurs when $D=E \cap T N$ and $\mathcal{E}=C^{\infty}(M)_{E}$ :

Proposition 2.3. Let $(M, P)$ be a Poisson manifold, $i_{N}: N \subset M a$ submanifold of $M$ and $E$ a subbundle of $\left.T M\right|_{N}$ as in Definition [2.1. If $\left\{C^{\infty}(M)_{E}, C^{\infty}(M)_{E}\right\} \subset C^{\infty}(M)_{E \cap T N}$ and $P^{\sharp}\left(E^{0}\right) \subset T N$, then $(M, P, N, E)$ is Poisson reducible.

The following result, that we will use later, relates the Poisson bivector $P$ with its reduction $P^{\prime}$. Its proof is straightforward. 
Lemma 2.4. Let $(M, P, N, E)$ be a quadruple satisfying conditions of Proposition [2.3, so that it is Poisson reducible to $\left(Q, P^{\prime}\right)$. Then,

$$
P\left(\widetilde{\pi^{*} \lambda}, \widetilde{\pi^{*} \eta}\right) \circ i_{N}=P^{\prime}(\lambda, \eta) \circ \pi
$$

and

$$
d \pi \circ P^{\sharp}\left(\widetilde{\pi^{*} \lambda}\right)=P^{\prime \sharp}(\lambda) \circ \pi,
$$

for any $\lambda, \eta \in \Omega^{1}(Q)$ and any extensions $\widetilde{\pi^{*} \lambda}, \widetilde{\pi^{*} \eta} \in \Omega^{1}(M)$ of $\pi^{*} \lambda, \pi^{*} \eta$ vanishing on $E$.

A particular and important case where the assumptions of Proposition 2.3 are satisfied is when a certain canonical action (i.e. preserving the Poisson structure) of a Lie group is given [13].

Proposition 2.5. Let $(M, P)$ be a Poisson manifold and consider a canonical action of a Lie group $G$ on $(M, P)$ admitting an $A d^{*}$-equivariant moment map $J: M \rightarrow \mathcal{G}^{*}\left(\mathcal{G}\right.$ is the Lie algebra of $G$ and $\mathcal{G}^{*}$ its dual). Suppose that $\mu \in \mathcal{G}^{*}$ is a regular value of $J$ and that the isotropy subgroup $G_{\mu}$ of $\mu$ for the coadjoint representation of $G$, acts freely and properly on $N_{\mu}:=J^{-1}(\mu)$. Consider the quotient $Q_{\mu}:=N_{\mu} / G_{\mu}$, the associated canonical projection $\pi_{\mu}: N_{\mu} \rightarrow Q_{\mu}$ and the inclusion map $i_{\mu}: N_{\mu} \subset M$ as well. Then $\left(Q_{\mu}, P_{\mu}\right)$ is a Poisson manifold, its Poisson structure being defined by

$$
\{f, h\}_{P_{\mu}} \circ \pi_{\mu}=\{F, H\}_{P} \circ i_{\mu},
$$

for any $f, h \in C^{\infty}\left(Q_{\mu}\right)$ and any extensions $F, H \in C^{\infty}(M)$ of $f \circ \pi_{\mu}, h \circ \pi_{\mu}$, respectively, with $d F$ and $d H$ vanishing on $E_{\mu}$, where $\left(E_{\mu}\right)_{p}=T_{p}(G \cdot p)$, for all $p \in N_{\mu}$, and $G \cdot p$ is the orbit of $G$ containing $p$.

In this case, one proves that $\left(E_{\mu} \cap T N_{\mu}\right)_{p}=T_{p}\left(G_{\mu} \cdot p\right)$, for all $p \in N_{\mu}$. Therefore, the distribution $E_{\mu} \cap T N_{\mu}$ is integrable and the leaves of the foliation it determines are the $G_{\mu}$-orbits in $N_{\mu}$. The set of leaves is the manifold $Q_{\mu}$ and the canonical projection $\pi_{\mu}$ is a submersion. The canonicity of the action and the fact that $E_{\mu}=P^{\sharp}\left(\left(T N_{\mu}\right)^{0}\right)$ holds, ensure the two remaining conditions of Proposition 2.3 are also satisfied.

\subsection{Extension to the Poisson quasi-Nijenhuis manifolds with background}

Poisson reduction can be used as a base for reducing any manifold of Poisson type by adding the conditions which are needed to reduce, in an appropriate way, the additional structure. In particular, Marsden-Ratiu Poisson reduction was used by Vaisman in [18] for proving a reduction procedure for Poisson-Nijenhuis manifolds. In [18], Poisson-Nijenhuis reduction by a group action was also derived. In the sequel, we will extend these results to the case of Poisson quasi-Nijenhuis manifolds with background, using the more general Falceto-Zambon reduction procedure. 
Definition 2.6. Let $(M, P, A, \phi, H)$ be a Poisson quasi-Nijenhuis manifold with background, $i_{N}: N \subset M$ a submanifold of $M$, and $E$ a vector subbundle of $\left.T M\right|_{N}$ such that assumptions of Definition 2.1 are satisfied. We say that $(M, P, A, \phi, H)$ is reducible if there exists a Poisson quasi-Nijenhuis structure with background $\left(P^{\prime}, A^{\prime}, \phi^{\prime}, H^{\prime}\right)$ on the reduced manifold $Q$, such that the tensors $P^{\prime}, A^{\prime}, \phi^{\prime}, H^{\prime}$ are the projections of $P, A, \phi, H$ on $Q$, i.e. $P$ and $P^{\prime}$ are related by equation (13), $A$ and $A^{\prime}$ are related by

$$
\left.d \pi \circ A\right|_{T N}=A^{\prime} \circ d \pi,
$$

where it is assumed that $\left.A\right|_{T N}$ is well defined, i.e. that $A(T N) \subset T N$, and $\phi, \phi^{\prime}$ and $H, H^{\prime}$ are related by

$$
\begin{aligned}
& i_{N}^{*} \phi=\pi^{*} \phi^{\prime}, \\
& i_{N}^{*} H=\pi^{*} H^{\prime} .
\end{aligned}
$$

Next theorem gives sufficient conditions for such a reduction to occur.

Theorem 2.7. Let $(M, P, A, \phi, H)$ be a Poisson quasi-Nijenhuis manifold with background, $i_{N}: N \subset M$ a submanifold of $M$ and $E$ a vector subbundle of $\left.T M\right|_{N}$ as in Definition [2.1. Assume that:

i) $\left\{C^{\infty}(M)_{E}, C^{\infty}(M)_{E}\right\} \subset C^{\infty}(M)_{E \cap T N}$;

ii) $P^{\sharp}\left(E^{0}\right) \subset T N$;

iii) $A(T N) \subset T N, A(E) \subset E$ and $\left.A\right|_{T N}$ sends projectable vector fields to projectable vector fields;

iv) $i_{N}^{*}\left(\imath_{X} \phi\right)=0=i_{N}^{*}\left(\imath_{X} H\right)$, for all $X \in \mathfrak{X}^{1}(M)$ such that $\left.X\right|_{N} \in \Gamma(E)$.

Then, the tensors $P, A, \phi, H$ project to tensors $P^{\prime}, A^{\prime}, \phi^{\prime}, H^{\prime}$ on $Q$, respectively, and $\left(Q, P^{\prime}, A^{\prime}, \phi^{\prime}, H^{\prime}\right)$ is a Poisson quasi-Nijenhuis manifold with background.

Proof. Lemmas 2.8 and 2.9 are needed. They are presented just after this proof. We will prove the existence of the projections $P^{\prime}, A^{\prime}, \phi^{\prime}, H^{\prime}$ satisfying all the conditions (44)-(7). We will denote by $X, Y$ arbitrary vector fields on $N$ which are projectable to vector fields $X^{\prime}=\pi_{*} X, Y^{\prime}=\pi_{*} Y$ on $Q$ and $\tilde{X}, \tilde{Y} \in \mathfrak{X}^{1}(M)$ will be arbitrary extensions of $X, Y$.

From Proposition 2.3, we know that $P$ projects to $P^{\prime}$. As for the tensor $A$, since $A(T N) \subset T N$, we can consider the $(1,1)$-tensor $\left.A\right|_{T N}: T N \rightarrow T N$. Also, since $\left.A\right|_{T N}$ sends projectable vector fields to projectable vector fields and $A(E \cap T N) \subset E \cap T N$, there exists a unique $(1,1)$-tensor $A^{\prime}: T Q \rightarrow T Q$ satisfying $\left.d \pi \circ A\right|_{T N}=A^{\prime} \circ d \pi$. Take now $\lambda, \eta \in \Omega^{1}(Q)$ and let $\widetilde{\pi^{*} \lambda}, \widetilde{\pi^{*} \eta} \in$ $\Omega^{1}(M)$ be any extensions of $\pi^{*} \lambda, \pi^{*} \eta$ vanishing on $E$. Since $A(E) \subset E$, $\widetilde{\pi^{*} \lambda} \circ A$ and $\widetilde{\pi^{*} \eta} \circ A$ are extensions of $\pi^{*}\left(\lambda \circ A^{\prime}\right)$ and $\pi^{*}\left(\eta \circ A^{\prime}\right)$ vanishing on 
$E$. Therefore, from equation (14) and the fact that $P$ and $A$ satisfy (44), we have that

$P^{\prime}\left(\lambda \circ A^{\prime}, \eta\right) \circ \pi=P\left(\widetilde{\pi^{*} \lambda} \circ A, \widetilde{\pi^{*} \eta}\right) \circ i_{N}=P\left(\widetilde{\pi^{*} \lambda}, \widetilde{\pi^{*} \eta} \circ A\right) \circ i_{N}=P^{\prime}\left(\lambda, \eta \circ A^{\prime}\right) \circ \pi$, and hence $P^{\prime}$ and $A^{\prime}$ also satisfy (41).

From assumption (iv) and the fact of $\phi$ and $H$ being closed, we conclude that these forms are both projectable to closed 3-forms $\phi^{\prime}$ and $H^{\prime}$ on $Q$ defined by (17) and (18). Moreover, an easy computation gives

$$
\begin{aligned}
& i_{N}^{*}\left(\imath_{\tilde{X} \wedge \tilde{Y}} \phi\right)=\pi^{*}\left(\imath_{X^{\prime} \wedge Y^{\prime}} \phi^{\prime}\right), \\
& i_{N}^{*}\left(\imath_{\tilde{X} \wedge \tilde{Y}} H\right)=\pi^{*}\left(\imath_{X^{\prime} \wedge Y^{\prime}} H^{\prime}\right) .
\end{aligned}
$$

Using Lemma 2.8 and equations (20) and (15), we get

$$
\begin{aligned}
\pi^{*}\left(\mathcal{C}_{P^{\prime}, A^{\prime}}(\lambda, \eta)\right) & =i_{N}^{*}\left(\mathcal{C}_{P, A}\left(\widetilde{\pi^{*} \lambda}, \widetilde{\pi^{*} \eta}\right)\right)=i_{N}^{*}\left(-\imath_{P^{\sharp}\left(\widetilde{\pi^{*} \lambda}\right) \wedge P^{\sharp}\left(\widetilde{\pi^{*} \eta} \eta\right.} H\right) \\
& =\pi^{*}\left(-\imath_{P^{\prime \sharp} \lambda \wedge P^{\sharp \sharp} \eta} H^{\prime}\right),
\end{aligned}
$$

and since $\pi^{*}$ is injective, we conclude that the concomitant of $P^{\prime}$ and $A^{\prime}$ satisfies (5).

We will now compute the torsion of $A^{\prime}$. From (16), we easily get

$$
\mathcal{N}_{A^{\prime}}\left(X^{\prime}, Y^{\prime}\right) \circ \pi=d \pi \circ \mathcal{N}_{\left.A\right|_{T N}}(X, Y) .
$$

Moreover, since $\left.d i_{N} \circ A\right|_{T N}=A \circ d i_{N}$, we have

$$
\mathcal{N}_{A}(\tilde{X}, \tilde{Y}) \circ i_{N}=d i_{N} \circ \mathcal{N}_{\left.A\right|_{T N}}(X, Y),
$$

and therefore

$$
\begin{aligned}
\mathcal{N}_{A^{\prime}}\left(X^{\prime}, Y^{\prime}\right) \circ \pi & =\left.d \pi \circ\left(\mathcal{N}_{A}(\tilde{X}, \tilde{Y})\right)\right|_{N} \\
& =\left.d \pi \circ\left(P^{\sharp}\left(\imath_{\tilde{X} \wedge \tilde{Y}} \phi+\imath_{A \tilde{X} \wedge \tilde{Y}} H+\imath_{\tilde{X} \wedge A \tilde{Y}} H\right)\right)\right|_{N} .
\end{aligned}
$$

Notice that the vector field $P^{\sharp}\left(\imath_{\tilde{X} \wedge \tilde{Y}} \phi+\imath_{A \tilde{X} \wedge \tilde{Y}} H+\imath_{\tilde{X} \wedge A \tilde{Y}} H\right)$, on $M$, is tangent to $N$. This is a direct consequence of assumptions (ii) and (iv). Now, from (19) and (20), and noticing that $A \tilde{X}, A \tilde{Y}$ are extensions of $\left.A\right|_{T N} X,\left.A\right|_{T N} Y$ and that these last ones project to $A^{\prime} X^{\prime}, A^{\prime} Y^{\prime}$, the 1-forms in (21) are extensions of $\pi^{*}\left(\imath_{X^{\prime} \wedge Y^{\prime}} \phi^{\prime}\right), \pi^{*}\left(\imath_{A^{\prime} X^{\prime} \wedge Y^{\prime}} H^{\prime}\right), \pi^{*}\left(\imath_{X^{\prime} \wedge A^{\prime} Y^{\prime}} H^{\prime}\right)$, that vanish on $E$. Therefore, we can use equation (15) to write (21) as

$$
\mathcal{N}_{A^{\prime}}\left(X^{\prime}, Y^{\prime}\right)=P^{\prime \sharp}\left(\imath_{X^{\prime} \wedge Y^{\prime}} \phi^{\prime}+\imath_{A^{\prime} X^{\prime} \wedge Y^{\prime}} H^{\prime}+\imath_{X^{\prime} \wedge A^{\prime} Y^{\prime}} H^{\prime}\right),
$$

which is equation (6) .

It remains to check (7). From (2) and the fact of $\phi$ and $\phi^{\prime}$ being closed, we have $d_{A} \phi=-d \imath_{A} \phi$ and $d_{A^{\prime}} \phi^{\prime}=-d \imath_{A^{\prime}} \phi^{\prime}$, and so, from Lemma 2.9, we get

$$
\pi^{*}\left(d_{A^{\prime}} \phi^{\prime}\right)=-d\left(\pi^{*}\left(\imath_{A^{\prime}} \phi^{\prime}\right)\right)=-d\left(i_{N}^{*}\left(\imath_{A} \phi\right)\right)=i_{N}^{*} d \mathcal{H}=\pi^{*} d \mathcal{H}^{\prime} .
$$

This completes the proof of the theorem. 
Lemma 2.8. Let $P$ be a Poisson bivector on $M$ and $A: T M \rightarrow T M a$ (1,1)-tensor. Let moreover $i_{N}: N \subset M$ be a submanifold of $M$ and $E$ a vector subbundle of $\left.T M\right|_{N}$ such that conditions i), ii) and iii) of Theorem 2.7 are satisfied, so that $P$ projects to a Poisson bivector $P^{\prime}$ on $Q$ and $A$ to $a(1,1)$-tensor $A^{\prime}: T Q \rightarrow T Q$. Then,

$$
\pi^{*}\left(\mathcal{C}_{P^{\prime}, A^{\prime}}(\lambda, \eta)\right)=i_{N}^{*}\left(\mathcal{C}_{P, A}\left(\widetilde{\pi^{*} \lambda}, \widetilde{\pi^{*} \eta}\right)\right)
$$

for all $\lambda, \eta \in \Omega^{1}(Q)$ and any extensions $\widetilde{\pi^{*} \lambda}, \widetilde{\pi^{*} \eta} \in \Omega^{1}(M)$ of $\pi^{*} \lambda, \pi^{*} \eta$ vanishing on $E$.

Proof. Take any projectable vector field $X \in \mathfrak{X}^{1}(N)$ and set $X^{\prime}=\pi_{*} X$. Using equation (14), we get

$$
d\left(P^{\prime}(\lambda, \eta)\right)\left(A^{\prime} X^{\prime}\right) \circ \pi=d\left(P\left(\widetilde{\pi^{*} \lambda}, \widetilde{\pi^{*} \eta}\right)\right)\left(A d i_{N} X\right) \circ i_{N}
$$

and

$$
d\left(P^{\prime}\left(\lambda, A^{\prime t} \eta\right)\right)\left(X^{\prime}\right) \circ \pi=d\left(P\left(\widetilde{\pi^{*} \lambda}, A^{t} \widetilde{\pi^{*} \eta}\right)\right)\left(d i_{N} X\right) \circ i_{N},
$$

where, in the last equality, we used the fact that $\pi^{*}\left(A^{\prime t} \eta\right)=i_{N}^{*}\left(A^{t} \widetilde{\pi^{*} \eta}\right)$, which is easily seen to be equivalent to equation (16). Moreover, using equation (15), we have

$$
\begin{aligned}
d\left(A^{\prime t} \lambda\right)\left(P^{\prime \sharp} \eta, X^{\prime}\right) \circ \pi & =d\left(\pi^{*}\left(A^{\prime t} \lambda\right)\right)\left(P^{\sharp}\left(\widetilde{\pi^{*} \eta}\right), X\right) \\
& =d\left(i_{N}^{*}\left(A^{t} \widetilde{\pi^{*} \lambda}\right)\right)\left(P^{\sharp}\left(\widetilde{\pi^{*} \eta}\right), X\right) \\
& =d\left(A^{t} \widetilde{\pi^{*} \lambda}\right)\left(P^{\sharp}\left(\widetilde{\pi^{*} \eta}\right), d i_{N} X\right) \circ i_{N},
\end{aligned}
$$

and, by a similar reasoning,

$$
d \eta\left(P^{\prime \sharp} \lambda, A^{\prime} X^{\prime}\right) \circ \pi=d\left(\widetilde{\pi^{*} \eta}\right)\left(P^{\sharp}\left(\widetilde{\pi^{*} \lambda}\right), A d i_{N} X\right) \circ i_{N} .
$$

Therefore, from (3), we obtain

$$
\begin{aligned}
& \pi^{*}\left(\mathcal{C}_{P^{\prime}, A^{\prime}}(\lambda, \eta)\right)(X)= \\
= & d\left(A^{\prime t} \lambda\right)\left(P^{\prime \sharp} \eta, X^{\prime}\right) \circ \pi-d\left(A^{\prime t} \eta\right)\left(P^{\prime \sharp} \lambda, X^{\prime}\right) \circ \pi+d \eta\left(P^{\prime \sharp} \lambda, A^{\prime} X^{\prime}\right) \circ \pi \\
& -d \lambda\left(P^{\prime \sharp} \eta, A^{\prime} X^{\prime}\right) \circ \pi-d\left(P^{\prime}\left(\lambda, A^{\prime t} \eta\right)\right)\left(X^{\prime}\right) \circ \pi+d\left(P^{\prime}(\lambda, \eta)\right)\left(A^{\prime} X^{\prime}\right) \circ \pi \\
= & i_{N}^{*}\left(\mathcal{C}_{P, A}\left(\widetilde{\pi^{*} \lambda}, \widetilde{\pi^{*} \eta}\right)\right)(X),
\end{aligned}
$$

which proves (22).

Lemma 2.9. Let $i_{N}: N \subset M$ be a submanifold of $M, \pi: N \rightarrow Q$ a submersion onto a manifold $Q, \phi$ and $H$ closed 3 -forms on $M$ and $A: T M \rightarrow T M$ $a(1,1)$-tensor satisfying $A(T N) \subset T N$. Suppose that $\phi, H$ and $A$ are projectable by $\pi$, i.e. there exist tensors $\phi^{\prime}, H^{\prime}$ and $A^{\prime}$ on $Q$ satisfying equations (16), (17) and (18). Then,

$$
i_{N}^{*}\left(\imath_{A} \phi\right)=\pi^{*}\left(\imath_{A^{\prime}} \phi^{\prime}\right)
$$


and

$$
i_{N}^{*} \mathcal{H}=\pi^{*} \mathcal{H}^{\prime},
$$

where $\mathcal{H}$ is given by equation (8) and $\mathcal{H}^{\prime}$ is given by the same equation with $H^{\prime}$ and $A^{\prime}$.

Proof. It is a straightforward computation.

When $H=0$, Theorem 2.7 gives a reduction procedure for Poisson quasiNijenhuis manifolds. If, moreover, $\phi=0$, we get a reduction theorem for Poisson-Nijenhuis manifolds which is a slightly more general version of the one derived in [18].

Now we will consider the special case of reduction by symmetries.

Proposition 2.10. Let $\left(M, P, G, J, \mu, N_{\mu}, E_{\mu}, Q_{\mu}, P_{\mu}\right)$ be as in Proposition 2.5. Let also $A: T M \rightarrow T M$ be a $(1,1)$-tensor and $\phi, H$ closed 3 -forms on $M$ such that $(M, P, A, \phi, H)$ is a Poisson quasi-Nijenhuis manifold with background and such that the following conditions hold:

(a) $d J \circ A=d J$ at any point of $N_{\mu}$;

(b) there exists an endomorphism $C$ of $\mathcal{G}$ such that $A \tilde{\xi}=\widetilde{C \xi}$, for all $\xi \in \mathcal{G}$, where $\tilde{\xi}$ denotes the fundamental vector field on $M$ associated with $\xi$ by the action of $G$;

(c) $A$ is $G$-invariant, i.e. $\mathcal{L}_{\tilde{\xi}} A=0$, for all $\xi \in \mathcal{G}$;

(d) $i_{\mu}^{*}\left(\imath_{\tilde{\xi}} \phi\right)=0=i_{\mu}^{*}\left(\imath_{\tilde{\xi}} H\right)$, for all $\xi \in \mathcal{G}$.

Then, $(M, P, A, \phi, H)$ reduces to a Poisson quasi-Nijenhuis manifold with background $\left(Q_{\mu}, P_{\mu}, A_{\mu}, \phi_{\mu}, H_{\mu}\right)$, where $A_{\mu}, \phi_{\mu}$ and $H_{\mu}$ are the projections of $A, \phi$ and $H$ on $Q_{\mu}$, respectively.

Proof. We only need to prove (iii) and (iv) of Theorem 2.7. Since $T_{p} N_{\mu}=$ ker $d J(p), \forall p \in N_{\mu}$, condition (a) above implies that $A\left(T N_{\mu}\right) \subset T N_{\mu}$. As for the inclusion $A\left(E_{\mu}\right) \subset E_{\mu}$, it follows from (b) and the fact that

$$
\left(E_{\mu}\right)_{p}=T_{p}(G \cdot p)=\{\tilde{\xi}(p): \xi \in \mathcal{G}\},
$$

for all $p \in N_{\mu}$. Moreover, condition (c) implies that $A$ sends projectable vector fields to projectable vector fields, and so condition (iii) of Theorem 2.7 holds. Finally, that condition (d) implies condition (iv) of Theorem 2.7 is an obvious consequence of equality (25).

This result contains the group action reduction for Poisson-Nijenhuis manifolds presented in [18], and gives also a group action reduction for Poisson quasi-Nijenhuis manifolds. 


\subsection{Reduction of generalized complex manifolds with back- ground}

Taking into account that a gc manifold with background is a special case of a $\mathrm{PqNb}$ manifold, we can refine Theorem 2.7 and construct a reduction procedure for gc manifolds with background as follows:

Theorem 2.11. Let $(M, \mathcal{J}, H)$ be a generalized complex manifold with background, with $\mathcal{J}:=(A, P, \sigma)$ given by (11), $i_{N}: N \subset M$ a submanifold of $M$ and $E$ a vector subbundle of $\left.T M\right|_{N}$ as in Definition 2.1. Suppose that conditions (i), (ii) and (iii) of Theorem 2.7 are satisfied and, moreover,

(a) $\sigma^{b}(T N) \subset E^{0}$;

(b) $i_{N}^{*}\left(\imath_{X} d \sigma\right)=0=i_{N}^{*}\left(\imath_{X} H\right)$, for all $X \in \mathfrak{X}^{1}(M)$ such that $\left.X\right|_{N} \in \Gamma(E)$.

Then, the tensors $P, A, \sigma, H$ project to tensors $P^{\prime}, A^{\prime}, \sigma^{\prime}, H^{\prime}$ on $Q$, respectively, and $\left(Q, \mathcal{J}^{\prime}, H^{\prime}\right)$ is a generalized complex manifold with background where $\mathcal{J}^{\prime}$ is the vector bundle map determined by $P^{\prime}, A^{\prime}, \sigma^{\prime}$ as in (11).

Proof. By Theorem 1.7, $(M, P, A, d \sigma, H)$ is a PqNb manifold and properties (4) and (5) of Theorem 1.6 hold. From Theorem 2.7 we get the PqNb manifold $\left(Q, P^{\prime}, A^{\prime}, \phi^{\prime}, H^{\prime}\right)$, where $P^{\prime}, A^{\prime}, \phi^{\prime}, H^{\prime}$ are the projections of $P, A, d \sigma, H$, respectively. On the other hand, from conditions (a) and (b) above, $\sigma$ projects to a 2-form $\sigma^{\prime}$ on $Q$. Therefore, we have $\phi^{\prime}=d \sigma^{\prime}$ and so the reduced $\mathrm{PqNb}$ manifold that we obtain is in fact $\left(Q, P^{\prime}, A^{\prime}, d \sigma^{\prime}, H^{\prime}\right)$. It remains to show that the tensors $P^{\prime}, A^{\prime}, \sigma^{\prime}, H^{\prime}$ satisfy properties (4) and (5) of Theorem 1.6. We start by noticing that a simple computation gives

$$
i_{N}^{*} \sigma_{A}=\pi^{*} \sigma_{A^{\prime}}^{\prime}
$$

Then, in particular, since $\sigma_{A}$ is antisymmetric, $\sigma_{A^{\prime}}^{\prime}$ also is. Moreover, using (26) and Lemma 2.9, we can write

$$
i_{N}^{*}\left(d \sigma_{A}+H-\imath_{A} d \sigma-\mathcal{H}\right)=\pi^{*}\left(d \sigma_{A^{\prime}}^{\prime}+H^{\prime}-\imath_{A^{\prime}} d \sigma^{\prime}-\mathcal{H}^{\prime}\right)
$$

and so property (4) of Theorem 1.6 holds. Finally, given any projectable vector field $X \in \mathfrak{X}^{1}(N)$, we have

$$
\begin{aligned}
A^{\prime 2}\left(\pi_{*} X\right) & =\pi_{*}\left(\left(\left.A\right|_{T N}\right)^{2} X\right)=-\pi_{*} X-\pi_{*}\left(P^{\sharp}\left(\sigma^{b}(X)\right)\right) \\
& =-\pi_{*} X-P^{\prime \sharp}\left(\sigma^{\prime}\left(\pi_{*} X\right)\right),
\end{aligned}
$$

which proves (5) of Theorem 1.6. In the last equality above, we used equation (15). In fact, $\sigma^{b}(X)$ is an extension of $\pi^{*}\left(\sigma^{\prime b}\left(\pi_{*} X\right)\right)$ which vanishes on E.

When $H=0$, we recover a slightly more general version of the reduction procedure for gc manifolds found by Vaisman in [19].

Now, we will use Proposition 2.10 to construct a group action reduction procedure for gc manifolds with background. 
Proposition 2.12. Let $\left(M, P, G, J, \mu, N_{\mu}, Q_{\mu}, P_{\mu}\right)$ be as in Proposition 2.5. Let also $\sigma$ be a 2-form, $A: T M \rightarrow T M$ a $(1,1)$-tensor and $H$ a closed 3 -form on $M$, such that $(M, \mathcal{J}, H)$ is a generalized complex manifold with background, where the vector bundle map $\mathcal{J}$ is determined by $P, \sigma, A$, as in (11). Suppose that conditions (a), (b) and (c) of Proposition 2.10 are satisfied and, moreover,

(i) the orbits of $G$ and the level sets of the moment map $J$ are $\sigma$-orthogonal;

(ii) $i_{\mu}^{*}\left(\imath_{\tilde{\xi}} d \sigma\right)=0=i_{\mu}^{*}\left(\imath_{\tilde{\xi}} H\right)$, for all $\xi \in \mathcal{G}$.

Then, the tensors $\sigma, A, H$ project to tensors $\sigma_{\mu}, A_{\mu}, H_{\mu}$ on $Q_{\mu}$, respectively, and $\left(Q, \mathcal{J}_{\mu}, H_{\mu}\right)$ is a generalized complex manifold with background, where the vector bundle map $\mathcal{J}_{\mu}$ is determined by $P_{\mu}, \sigma_{\mu}, A_{\mu}$, as in (11).

Proof. Assumption (i) guarantees condition (a) of Theorem 2.11, All the remaining conditions of that theorem are satisfied (see the proof of Proposition 2.10).

Remark 2.13. There are several different approaches to reduction of generalized complex structures (without background). In [16], the reduction of a gc structure $\mathcal{J}$ on a manifold $M$ is performed by the action of a Lie group $G$ on $M$. The action should preserve $\mathcal{J}$ and a $G$-invariant submanifold $N$ of $M$, where $G$ acts free and properly, is taken. The authors obtain sufficient conditions to $\mathcal{J}$ descend to the quotient $N / G$. The procedure consists in reducing the complex Dirac structures on $M$ that determine $\mathcal{J}$, i.e. their $( \pm i)$-eigenbundles, to Dirac structures on $N / G$ that are going to define the reduced gc structure.

In [3], the reduction of gc structures (with background) is also based on Dirac reduction, but with a different approach. Dirac reduction is derived from an exact Courant algebroid reduction procedure which involves the concept of an "extended action" and its associated moment map.

In [10], the authors introduce the concept of generalized moment map for a compact Lie group action on a generalized complex manifold and then use this notion to implement reduction, i.e. to define a generalized complex structure on the reduced space. In an appendix of the paper, this approach is extended to generalized complex structures with background.

In [20], the reduction of an exact Courant algebroid $E \rightarrow M$ is performed without any group action. Instead, a coisotropic subbundle $K \rightarrow C$ ( $C$ is a submanifold of $M$ ) of the exact Courant algebroid $E$ is used to obtain a reduced exact Courant algebroid $\underline{E} \rightarrow \underline{C}$. The author takes a gc structure $\mathcal{J}$ on the exact Courant algebroid $E$ and gives sufficient conditions on $\mathcal{J}$, $K$ and $C$ to $\mathcal{J}$ descend to a reduced gc structure $\underline{\mathcal{J}}$ on $\underline{E} \rightarrow \underline{C}$. 


\section{Gauge transformations of Poisson quasi-Nijenhuis structures with background}

\subsection{Definition}

An important concept in generalized complex geometry is that of gauge transformation. As shown by Gualtieri [7, given a closed 3-form $H$ and a 2 -form $B$ on $M$, the mapping

$$
\text { B : } X+\alpha \mapsto X+\alpha+\imath_{X} B
$$

is a vector bundle automorphism of $\mathbb{T} M$ which is compatible with Courant brackets with backgrounds $H$ and $H+d B$, i.e.

$$
\mathbf{B}[X+\alpha, Y+\beta]_{H}=[\mathbf{B}(X+\alpha), \mathbf{B}(Y+\beta)]_{H+d B} .
$$

The mapping $\mathbf{B}$ is called a $B$-field or a gauge transformation and its matrix representation is given by

$$
\mathbf{B}=\left(\begin{array}{cc}
\mathrm{Id} & 0 \\
B^{b} & \mathrm{Id}
\end{array}\right)
$$

It acts on gc structures with background $H$ by the invertible map $\mathcal{J} \mapsto$ $\mathbf{B}^{-1} \mathcal{J} \mathbf{B}$ and, as it was remarked in [7], $\mathbf{B}^{-1} \mathcal{J} \mathbf{B}$ is a gc structure with background $H+d B$. If $\mathcal{J}$ is given by (11), then

$$
\mathbf{B}^{-1} \mathcal{J} \mathbf{B}=\left(\begin{array}{cc}
A+P^{\sharp} B^{b} & P^{\sharp} \\
\sigma^{b}-B^{b} P^{\sharp} B^{b}-B^{b} A-A^{t} B^{b} & -A^{t}-B^{b} P^{\sharp}
\end{array}\right),
$$

so that the Poisson bivector $P$ is preserved, the $(1,1)$-tensor $A$ is replaced by $A+P^{\sharp} B^{b}$, and the 2 -form $\sigma$ goes to the 2 -form $\tilde{\sigma}$ given by

$$
\tilde{\sigma}=\sigma-B_{C}-\imath_{A} B,
$$

where $C$ is the $(1,1)$-tensor $P^{\sharp} B^{b}$ and $B_{C}$ is the 2-form given by $B_{C}(X, Y)=$ $B(C X, Y)$.

Having in mind that a gc structure with background is a special case of a $\mathrm{PqNb}$ structure, we now extend the concept of gauge transformation to the latter.

Theorem 3.1. Let $(P, A, \phi, H)$ be a Poisson quasi-Nijenhuis structure with background on $M$, and $B \in \Omega^{2}(M)$. Consider the tensors $\tilde{P}, \tilde{A}, \tilde{\phi}, \tilde{H}$ on $M$ given by:

$$
\begin{aligned}
& \tilde{P}=P, \\
& \tilde{A}=A+P^{\sharp} B^{b}, \\
& \tilde{\phi}=\phi-d B_{C}-d\left(\imath_{A} B\right), \\
& \tilde{H}=H+d B .
\end{aligned}
$$

Then, $(\tilde{P}, \tilde{A}, \tilde{\phi}, \tilde{H})$ is a Poisson quasi-Nijenhuis structure with background on $M$. 
In order to prove the theorem, we need some lemmas. Their proofs are included in the Appendix.

Lemma 3.2. Let $P$ be a Poisson bivector on $M$ and $B \in \Omega^{2}(M)$. Consider the $(1,1)$-tensor $C=P^{\sharp} B^{b}$. Then, the concomitant of $P$ and $C$ is given by

$$
\mathcal{C}_{P, C}(\alpha, \beta)=-\imath_{P^{\sharp} \alpha \wedge P^{\sharp} \beta} d B,
$$

for all $\alpha, \beta \in \Omega^{1}(M)$, and the torsion of $C$ reads

$$
\mathcal{N}_{C}(X, Y)=P^{\sharp}\left(\imath_{C X \wedge Y} d B+\imath_{X \wedge C Y} d B-\imath_{X \wedge Y} d B_{C}\right),
$$

for all $X, Y \in \mathfrak{X}^{1}(M)$. Moreover, we have

$$
d_{C} B_{C}=\mathcal{B}^{C, C}-d B_{C^{2}}
$$

where, for any $(1,1)$-tensors $S, T$, we denote

$$
\mathcal{B}^{S, T}(X, Y, Z)=\circlearrowleft_{X, Y, Z} d B(S X, T Y, Z),
$$

and $B_{C^{2}}$ is the 2-form defined by $B_{C^{2}}(X, Y)=B\left(C^{2} X, Y\right)$.

Lemma 3.3. Take tensors $Q \in \mathfrak{X}^{2}(M), H \in \Omega^{3}(M)$ and $A \in \operatorname{End}(T M)$ such that

$$
Q^{\sharp} A^{t}=A Q^{\sharp},
$$

and

$$
\mathcal{C}_{Q, A}(\alpha, \beta)=-\imath_{Q^{\sharp} \alpha \wedge Q^{\sharp} \beta} H,
$$

for all $\alpha, \beta \in \Omega^{1}(M)$. Take also $B \in \Omega^{2}(M)$ and denote $C=Q^{\sharp} B^{b}$. Then, we have

$$
\begin{gathered}
{[A X, C Y]-A[C X, Y]-A[X, C Y]+A C[X, Y]} \\
+[C X, A Y]-C[A X, Y]-C[X, A Y]+C A[X, Y]= \\
Q^{\sharp}\left(\imath_{A X \wedge Y} d B+\imath_{X \wedge A Y} d B-\imath_{X \wedge Y} d\left(\imath_{A} B\right)+\imath_{C X \wedge Y} H+\imath_{X \wedge C Y} H\right),
\end{gathered}
$$

for all $X, Y \in \mathfrak{X}^{1}(M)$, and, moreover,

$$
d_{A} B_{C}+d_{C}\left(\imath_{A} B\right)=\mathcal{H}^{C, C}+\mathcal{B}^{A, C}+\mathcal{B}^{C, A}-d B_{A C}-d\left(\imath_{C A} B\right),
$$

where, for any $(1,1)$-tensors $S, T$, we denote

$$
\mathcal{H}^{S, T}(X, Y, Z)=\circlearrowleft_{X, Y, Z} H(S X, T Y, Z),
$$

and $B_{A C}$ is the 2-form defined by $B_{A C}(X, Y)=B(A C X, Y)$. 
Lemma 3.4. Take tensors $Q \in \mathfrak{X}^{2}(M), \phi, H \in \Omega^{3}(M)$, and $A \in \operatorname{End}(T M)$, and suppose that

$$
\mathcal{N}_{A}(X, Y)=Q^{\sharp}\left(\imath_{X \wedge Y} \phi+\imath_{A X \wedge Y} H+\imath_{X \wedge A Y} H\right),
$$

for all $X, Y \in \mathfrak{X}^{1}(M)$. Take also $B \in \Omega^{2}(M)$ and denote $C=Q^{\sharp} B^{b}$. Then, we have that

$$
d_{A}\left(\imath_{A} B\right)=\mathcal{H}^{A, C}+\mathcal{H}^{C, A}+\mathcal{B}^{A, A}-d B_{A, A}+\imath_{C} \phi,
$$

where $B_{A, A}$ is the 2-form given by $B_{A, A}(X, Y)=B(A X, A Y)$.

Proof of Theorem 3.1. Let us show that $(\tilde{P}, \tilde{A}, \tilde{\phi}, \tilde{H})$ satisfies conditions (44)(7). We have

$$
\tilde{A} \tilde{P}^{\sharp}=\left(A+P^{\sharp} B^{b}\right) P^{\sharp}=P^{\sharp} A^{t}+P^{\sharp}\left(P^{\sharp} B^{b}\right)^{t}=\tilde{P}^{\sharp} \tilde{A}^{t},
$$

which is (44). Condition (5) follows from (35):

$\mathcal{C}_{\tilde{P}, \tilde{A}}(\alpha, \beta)=\mathcal{C}_{P, A}(\alpha, \beta)+\mathcal{C}_{P, C}(\alpha, \beta)=-\imath_{P^{\sharp} \alpha \wedge P^{\sharp} \beta}(H+d B)=-\imath_{\tilde{P}^{\sharp} \alpha \wedge \tilde{P} \sharp \beta} \tilde{H}$,

for all $\alpha, \beta \in \Omega^{1}(M)$. To compute the torsion of $\tilde{A}$ we use (36) and (40):

$$
\begin{aligned}
\mathcal{N}_{\tilde{A}}( & X, Y)=\mathcal{N}_{A}(X, Y)+\mathcal{N}_{C}(X, Y) \\
& +([A X, C Y]-A[C X, Y]-A[X, C Y]+A C[X, Y]) \\
& +([C X, A Y]-C[A X, Y]-C[X, A Y]+C A[X, Y]) \\
= & P^{\sharp}\left(\imath_{X \wedge Y} \phi+\imath_{A X \wedge Y} H+\imath_{X \wedge A Y} H+\imath_{C X \wedge Y} d B+\imath_{X \wedge C Y} d B-\imath_{X \wedge Y} d B_{C}\right) \\
& +P^{\sharp}\left(\imath_{A X \wedge Y} d B+\imath_{X \wedge A Y} d B-\imath_{X \wedge Y} d\left(\imath_{A} B\right)+\imath_{C X \wedge Y} H+\imath_{X \wedge C Y} H\right) \\
= & \tilde{P}^{\sharp}\left(\imath_{X \wedge Y} \tilde{\phi}+\imath_{\tilde{A} X \wedge Y} \tilde{H}+\imath_{X \wedge \tilde{A} Y} \tilde{H}\right),
\end{aligned}
$$

for all $X, Y \in \mathfrak{X}^{1}(M)$. Finally, from (37), (41) and (43), together with the identity $d \circ d_{A}=-d_{A} \circ d$, we get

$$
\begin{aligned}
d_{\tilde{A}} \tilde{\phi} & =d_{A} \phi-d_{A} d B_{C}-d_{A} d\left(\imath_{A} B\right)+d_{C} \phi-d_{C} d B_{C}-d_{C} d\left(\imath_{A} B\right) \\
& =d \mathcal{H}^{A, A}+d \mathcal{H}^{C, C}+d \mathcal{H}^{A, C}+d \mathcal{H}^{C, A}+d \mathcal{B}^{A, A}+d \mathcal{B}^{C, C}+d \mathcal{B}^{A, C}+d \mathcal{B}^{C, A} \\
& =d \tilde{\mathcal{H}},
\end{aligned}
$$

where $\tilde{\mathcal{H}}$ is the 3 -form given by $\tilde{\mathcal{H}}(X, Y, Z)=\circlearrowleft_{X, Y, Z} \tilde{H}(\tilde{A} X, \tilde{A} Y, Z)$, for all $X, Y, Z \in \mathfrak{X}^{1}(M)$. This completes the proof.

Let $\mathfrak{C}_{P q N b}(M)$ denote the class of all Poisson quasi-Nijenhuis structures with background on $M$. 
Definition 3.5. Let $B$ be a 2-form on $M$. The map $\mathfrak{B}: \mathfrak{C}_{P q N b}(M) \rightarrow$ $\mathfrak{C}_{P q N b}(M)$ which assigns to each PqNb structure $(P, A, \phi, H) \in \mathfrak{C}_{P q N b}(M)$ the PqNb structure $(\tilde{P}, \tilde{A}, \tilde{\phi}, \tilde{H})$ defined by equations (31)-(34) is called the gauge transformation on $\mathfrak{C}_{P q N b}(M)$ determined by $B$.

Example 3.6. Take the PqNb structure $(P, A, \phi, H)$ on $\mathbb{R}^{3}$ of Example 1.2. The gauge transformation of this structure determined by the 2 -form $B=d x_{2} \wedge d x_{3}$ is the PqNb structure $(\tilde{P}, \tilde{A}, \tilde{\phi}, \tilde{H})$ with $\tilde{P}=P, \tilde{A}=A+$ $f \frac{\partial}{\partial x_{1}} \otimes d x_{3}, \tilde{\phi}=\phi$ and $\tilde{H}=H$. In this case only the $(1,1)$-tensor is modified but $\mathcal{C}_{\tilde{P}, \tilde{A}}=\mathcal{C}_{P, A}$.

Remark 3.7. Notice that the gauge transformation of gc structures with background, defined by (30), can be recovered from Theorem 3.1 if we additionally specify the transformation of the 2 -form $\sigma$. Moreover, using the alternative definition of gc structure with background in Remark 1.5, a gauge transformation of a gc structure $L$, with background $H$, is a new gc structure $L^{\prime}$ with background $H+d B$, obtained from the action of a (real) 2-form $B$ on $L, L^{\prime}=\mathbf{B} L$, with $\mathbf{B}$ given by (29).

Gauge transformations can preserve main subclasses of PqNb structures on $M$. In fact, if we require $B$ to be closed, then the associated gauge transformation will preserve the class of $\mathrm{PqN}$ structures. Moreover, given a PN structure $(P, A)$, if $B$, besides being closed, satisfies $d\left(B_{C}+\imath_{A} B\right)=0$, then $\mathfrak{B}(P, A)=\left(P, A+P^{\sharp} B^{b}\right)$ is still a PN structure.

Example 3.8. Consider the PN structure on $\mathbb{R}^{3}$ defined by $P=\frac{\partial}{\partial x_{1}} \wedge \frac{\partial}{\partial x_{2}}$ and $A=e^{x_{3}}\left(\frac{\partial}{\partial x_{1}} \otimes d x_{1}+\frac{\partial}{\partial x_{2}} \otimes d x_{2}+\frac{\partial}{\partial x_{3}} \otimes d x_{3}+x_{2} \frac{\partial}{\partial x_{2}} \otimes d x_{3}\right)$ and take the 2 -form $B=e^{x_{2}} d x_{2} \wedge d x_{3}$. We have $C=e^{x_{2}} \frac{\partial}{\partial x_{1}} \otimes d x_{3}$ and $\imath_{A} B=$ $2 e^{x_{3}} B$. Thus, $d B=0, B_{C}=0$ and $d\left(\imath_{A} B\right)=0$, and therefore, the gauge transformation of the initial PN structure is still a PN structure.

Remark 3.9. Theorem 3.1 and Definition 3.5 can be straightforwardly generalized for a generic Lie algebroid $E$ over $M$. In fact, all the computations made to prove Theorem 3.1 and Lemmas 3.2 3.4 are still valid in such case. So, if $\mathfrak{C}_{P q N b}(E)$ denotes the class of all PqNb structures on $E$ and a 2-form $B$ on $E$ is given, we define the associated gauge transformation $\mathfrak{B}: \mathfrak{C}_{P q N b}(E) \rightarrow \mathfrak{C}_{P q N b}(E)$ by setting $\mathfrak{B}(P, A, \phi, H)=(\tilde{P}, \tilde{A}, \tilde{\phi}, \tilde{H})$ where $\tilde{P}=P, \tilde{A}=A+C, \tilde{\phi}=\phi-d_{E} B_{C}-d_{E}\left(\imath_{A} B\right)$ and $\tilde{H}=H+d_{E} B$, with $C=P^{\sharp} B^{b}$.

Remark 3.10. It is worth to mention that the expression gauge transformation is used in literature, by some authors, with a different meaning from that in Definition 3.5. We will point out one big difference. B-field operation 
(or gauge transformation) defined by (27) was used in [14, 7, 2] to transform Dirac structures of $\mathbb{T} M$ and, due to its own properties, a gauge transformation of a Dirac structure is still a Dirac structure (eventually with respect to a different Courant bracket on $\mathbb{T} M$ ). As it is well known, Poisson structures can be viewed as Dirac subbundles; more precisely, if $P$ is a Poisson bivector on $M$, then its graph $L_{P}$ is a Dirac structure of $\mathbb{T} M$. However, the image of $L_{P}$ under the mapping (27), which is a Dirac structure, is not, in general, the graph of a Poisson bivector [14]. Under some mild conditions this could happen and, if this is the case, the new Poisson tensor is different from the initial one. The philosophy in this paper is quite different since, according to Theorem 3.1, the Poisson bivector in a PqNb structure does not change under gauge transformations.

\subsection{Construction of Poisson quasi-Nijenhuis structures with background}

Gauge transformations can be used as a tool for generating PqNb structures from other $\mathrm{PqNb}$ structures but also to construct richer examples from simpler ones. For example, we can construct $\mathrm{PqNb}$ structures from a Poisson bivector $P$, since any Poisson structure can be viewed as a $\mathrm{PqNb}$ structure where $A, \phi$ and $H$ vanish. In fact, according to Definition 3.5, given a 2-form $B$ on $M$, the associated gauge transformation takes a Poisson structure $P$ to the $\mathrm{PqNb}$ structure $\left(P, C,-d B_{C}, d B\right)$. This proves the following:

Theorem 3.11. Let $P$ be a Poisson bivector on $M$ and $B \in \Omega^{2}(M)$. Consider the $(1,1)$-tensor $C=P^{\sharp} B^{b}$. Then, $\left(P, C,-d B_{C}, d B\right)$ is a Poisson quasi-Nijenhuis structure with background on $M$.

According to this theorem, we are able to construct PqNb structures from any given 2-form on a Poisson manifold. This result was also derived by Antunes in [1] using the supergeometric techniques. Theorem 3.11] is also valid for a generic Lie algebroid $E$ over $M$ (see Remark 3.9) and this was in fact the approach followed in [1].

We may now ask whether it is possible to choose a Poisson bivector $P$ on $M$ and $B \in \Omega^{2}(M)$ in such a way that $\mathcal{J}:=\left(C, P,-B_{C}\right)$ is a gc structure with background $d B$, i.e. $\left(P, C,-d B_{C}, d B\right)$ is a $\mathrm{PqNb}$ structure and conditions (4) and (5) of Theorem [1.6 hold. The answer is no. If $\mathcal{J}$ was a gc structure with background $d B$, then we would have $C^{2}=-\mathrm{Id}-$ $P^{\sharp}\left(-B_{C}\right)^{b}=-\mathrm{Id}+C^{2}$ and this is an impossible condition.

However, if (and only if) we can choose $P$ nondegenerate, there is one, and only one, closed 2 -form $\omega$ that we can add to $-B_{C}$ in order that $\mathcal{J}^{\prime}:=$ $\left(C, P,-B_{C}+\omega\right)$ is a gc structure with background $d B$. This 2-form $\omega$ is the symplectic form associated to $P$, i.e. $\omega^{b}=-\left(P^{\sharp}\right)^{-1}$. In fact, in this case, $\mathcal{J}^{\prime}$ is the image, by the gauge transformation determined by $B$, of the gc 
structure $\mathcal{J}_{\text {sympl }}:=(0, P, \omega)$ and therefore is a gc structure with background $d B$.

If the 2 -form $B$ in Theorem 3.11 additionally satisfies

$$
\imath_{P^{\sharp} \alpha \wedge P^{\sharp} \beta} d B=0,
$$

for all $\alpha, \beta \in \Omega^{1}(M)$, then it is easy to see that the contribution of the background $d B$ in equations (5)-(17) vanishes, i.e.,

$$
\mathcal{C}_{P, C}(\alpha, \beta)=0 \quad, \quad \mathcal{N}_{C}(X, Y)=P^{\sharp}\left(\imath_{X \wedge Y}\left(-d B_{C}\right)\right) \quad, \quad d_{C}\left(-d B_{C}\right)=0 .
$$

We have therefore the following result:

Theorem 3.12. Let $P$ be a Poisson bivector on $M$ and $B$ a 2-form on $M$ satisfying (44). Then, $\left(P, C,-d B_{C}\right)$ is a Poisson quasi-Nijenhuis structure on $M$. In particular, this is true when $d B=0$.

This theorem gives a way of constructing PqN structures from a 2-form on a Poisson manifold. Moreover, in its version for a generic Lie algebroid $E$ over $M$, this result contains Theorem 3.2 in [17]. Just notice that, when

$$
\imath_{P^{\sharp} \alpha} d_{E} B=0,
$$

for all $\alpha \in \Gamma\left(E^{*}\right)$, we have $\imath_{C} d_{E} B=0$ and therefore

$$
[B, B]_{P}=2 \imath_{C} d_{E} B-2 d_{E} B_{C}=-2 d_{E} B_{C} .
$$

So, when (45) holds and $[B, B]_{P}=0$, the pair $(P, C)$ is a PN structure on $E$. Notice also that we do not need the anchor of $E$ to be injective as it was required in [17.

So far, we have used gauge transformations to construct $\mathrm{PqNb}$ and $\mathrm{PqN}$ structures from simpler ones. But we can also use gauge transformations in the opposite way, i.e. to get simpler structures from richer ones. For example, given a $\mathrm{PqNb}$ structure $(P, A, \phi, H)$ on $M$ with $H$ exact, we can choose $B \in \Omega^{2}(M)$ such that $H=d B$ and then consider the gauge transformation associated with $-B$, which takes $(P, A, \phi, H)$ to the PqN structure $\left(P, A-C, \phi-d B_{C}+d\left(\imath_{A} B\right)\right)$. By imposing additional restrictions on $B$, we may obtain a PN structure or even a Poisson one. Also, by considering gauge transformations associated with closed 2-forms, we are able to turn $\mathrm{PqN}$ structures into PN or even Poisson structures.

Next, we will show that more examples of PqNb structures can be constructed if we combine conformal change with gauge transformation. First, notice that if $P$ is a Poisson bivector on $M$ and $f \in C^{\infty}(M)$ is a Casimir of $P$, then $e^{f} P$ is a Poisson tensor:

$$
\left[e^{f} P, e^{f} P\right]=e^{f}\left(2\left[P, e^{f}\right] \wedge P+e^{f}[P, P]\right)=0 .
$$


The bivector $P^{\prime}=e^{f} P$ is called the conformal change of $P$ by $e^{f}$.

Take a Poisson bivector $P$ on $M$, a Casimir $f \in C^{\infty}(M)$ of $P$ and a 2-form $B$ on $M$. According to Theorem 3.11, $\left(P, C,-d B_{C}, d B\right)$, with $C=P^{\sharp} B^{b}$, is a $\mathrm{PqNb}$ structure on $M$, which is obtained from the Poisson tensor $P$ by the gauge transformation determined by $B$. Consider now the Poisson bivector $P^{\prime}=e^{f} P$ and the 2-form $B^{\prime}=e^{-f} B$. Applying again Theorem 3.11, we get a new $\mathrm{PqNb}$ structure on $M,\left(P^{\prime}, C^{\prime},-d B_{C^{\prime}}^{\prime}, d B^{\prime}\right)$, which is related to $\left(P, C,-d B_{C}, d B\right)$ by the formulae:

$$
P^{\prime}=e^{f} P ; C^{\prime}=C ; d B_{C^{\prime}}^{\prime}=e^{-f}\left(d B_{C}-d f \wedge B_{C}\right) ; d B^{\prime}=e^{-f}(d B-d f \wedge B) .
$$

We see that the $(1,1)$-tensor $C$ is fixed, while all the other tensors change. However, if we wish, we may fix the background of the $\mathrm{PqNb}$ structure. It suffices to apply Theorem 3.11 to the Poisson tensor $P^{\prime}=e^{f} P$ and the 2form $B^{\prime}=B$. In this case, the $(1,1)$-tensor $C=P^{\sharp} B^{b}$ changes to $C^{\prime}=e^{f} C$ and $d B_{C^{\prime}}^{\prime}=e^{f}\left(d B_{C}+d f \wedge B_{C}\right)$.

Summarizing, we have proved the following:

Proposition 3.13. Let $P$ be a Poisson bivector on $M, f \in C^{\infty}(M)$ a Casimir of $P$ and $B$ a 2-form on $M$. Consider the $(1,1)$-tensor $C=P^{\sharp} B^{b}$. Then,

$$
\left(e^{f} P, C, e^{-f}\left(-d B_{C}+d f \wedge B_{C}\right), e^{-f}(d B-d f \wedge B)\right)
$$

and

$$
\left(e^{f} P, e^{f} C, e^{f}\left(-d B_{C}-d f \wedge B_{C}\right), d B\right)
$$

are Poisson quasi-Nijenhuis structures with background on $M$.

\subsection{Some properties of gauge transformations}

Let us now consider the set Gauge $(M)$ of all gauge transformations on $\mathfrak{C}_{P q N b}(M)$ and denote by $\mathfrak{G}: \Omega^{2}(M) \rightarrow \operatorname{Gauge}(M)$ the map which assigns to each 2-form $B$ on $M$ the gauge transformation $\mathfrak{B}$ associated with $B$, i.e. $\mathfrak{B}=\mathfrak{G}(B)$. We can give $\operatorname{Gauge}(M)$ a natural group structure as follows:

Theorem 3.14. The set Gauge $(M)$ is an abelian group under the composition of maps, the identity element being the gauge transformation associated with the zero 2-form, and the inverse of $\mathfrak{G}(B)$ being $\mathfrak{G}(-B)$, for all $B \in \Omega^{2}(M)$. Moreover, the map $\mathfrak{G}$ is a group isomorphism from the abelian group $\left(\Omega^{2}(M),+\right)$ into $(\operatorname{Gauge}(M), \circ)$.

Proof. Given any $B_{1}, B_{2} \in \Omega^{2}(M)$, the composition of the associated gauge transformations is given by $\left(\mathfrak{G}\left(B_{1}\right) \circ \mathfrak{G}\left(B_{2}\right)\right)(P, A, \phi, H)=(\hat{P}, \hat{A}, \hat{\phi}, \hat{H})$ where

$$
\begin{aligned}
& \hat{P}=P \\
& \hat{A}=A+C_{1}+C_{2} \\
& \hat{\phi}=\phi-d B_{2 C_{2}}-d\left(\imath_{A} B_{2}\right)-d B_{1 C_{1}}-d\left(\imath_{A} B_{1}\right)-d\left(\imath_{C_{2}} B_{1}\right) \\
& \hat{H}=H+d B_{1}+d B_{2}
\end{aligned}
$$


with $C_{i}=P^{\sharp} B_{i}^{b}, i=1,2$. Since $B_{2}\left(C_{1} X, Y\right)=B_{1}\left(X, C_{2} Y\right)$, for all $X, Y \in$ $\mathfrak{X}^{1}(M)$, we can write (46) as

$$
\hat{\phi}=\phi-d\left(B_{1}+B_{2}\right)_{C_{1}+C_{2}}-d \imath_{A}\left(B_{1}+B_{2}\right),
$$

and so we realize that the composition $\mathfrak{G}\left(B_{1}\right) \circ \mathfrak{G}\left(B_{2}\right)$ is indeed the gauge transformation associated with $B_{1}+B_{2}$, i.e.

$$
\mathfrak{G}\left(B_{1}+B_{2}\right)=\mathfrak{G}\left(B_{1}\right) \circ \mathfrak{G}\left(B_{2}\right) .
$$

From this relation, the proof of the first part of the theorem is obvious and this same relation means that $\mathfrak{G}$ is a group homomorphism. Since by definition $\mathfrak{G}$ is a surjection, it just remains to prove that it is an injection. Take $B \in \Omega^{2}(M)$ and suppose that $\mathfrak{G}(B)=$ Id. Then, applying $\mathfrak{G}(B)$ on $\mathrm{PqNb}$ structures of the form $(P, 0,0,0)$, we see that $P^{\sharp} B^{b}=0$ for all Poisson bivectors $P$ on $M$. Therefore, we must have $B=0$. In fact, for any point $m \in M$, we can find local coordinates around $m$, and a bump function on $M$ which is nonzero at $m$, and prove that if $B \neq 0$, we can construct a Poisson tensor $P$ such that $P^{\sharp} B^{b} \neq 0$.

We conclude, from Theorem 3.14, that there exists a group action of $\Omega^{2}(M)$ on $\mathfrak{C}_{P q N b}(M)$, given by

$$
\begin{aligned}
\Omega^{2}(M) \times \mathfrak{C}_{P q N b}(M) & \rightarrow \mathfrak{C}_{P q N b}(M) \\
(B,(P, A, \phi, H)) & \mapsto\left(P, A+C, \phi-d B_{C}-d\left(\imath_{A} B\right), H+d B\right) .
\end{aligned}
$$

Two elements of $\mathfrak{C}_{P q N b}(M)$ are said to be gauge equivalent if they lie in the same orbit. All equivalent $\mathrm{PqNb}$ structures on $M$ have the same Poisson tensor. However, from the results of the previous section, one single orbit may contain different types of structures, i.e we can have gauge equivalence between Poisson and $\mathrm{PqNb}$ structures, between $\mathrm{PqN}$ and $\mathrm{PqNb}$, and so on. In the case of a nondegenerate Poisson bivector we derive the following:

Proposition 3.15. Given a nondegenerate Poisson bivector $P$ on $M$, the set of all PqNb structures having $P$ as the associated Poisson bivector is the $\Omega^{2}(M)$-orbit of the Poisson structure $(P, 0,0,0)$. In other words, these $P q N b$ structures are all those of the form $\left(P, P^{\sharp} B^{b},-d B_{P^{\sharp} B^{b}}, d B\right)$ with $B \in$ $\Omega^{2}(M)$.

Proof. Let $(P, A, \phi, H)$ be a $\mathrm{PqNb}$ structure where $P$ is nondegenerate. Because $P^{\sharp}$ is invertible, $\phi$ and $H$ are the unique 3 -forms satisfying equations (5) and (6) for $P$ and $A$. On the other hand, as a consequence of equation (4), the (0,2)-tensor $B$ defined by $B^{b}=\left(P^{\sharp}\right)^{-1} A$ is antisymmetric and therefore we can write $A=P^{\sharp} B^{b}$ with $B \in \Omega^{2}(M)$. Moreover, the gauge transformation associated with $B$ of the Poisson structure $(P, 0,0,0)$ is $\left(P, P^{\sharp} B^{b},-d B_{P^{\sharp} B^{b}}, d B\right)$. Therefore, since the 3 -forms $\phi$ and $H$ are unique, we must have $\phi=-d B_{P^{\sharp} B^{b}}$ and $H=d B$. This proves the result. 
In particular, we have seen that, given a nondegenerate Poisson bivector $P$ and a (1,1)-tensor $A$ satisfying equation (4), we have one and only one $\mathrm{PqNb}$ structure of the form $(P, A, \cdot, \cdot)$. For degenerate Poisson bivectors, this is not in general true. For example, given a degenerate Poisson bivector $P$ on $M$ and $B \in \Omega^{2}(M),\left(P, P^{\sharp} B^{b},-d B_{P^{\sharp} B^{b}}, d B\right)$ is a PqNb structure and $\left(P, P^{\sharp} B^{b},-d B_{P^{\sharp} B^{b}}, d B+H\right)$ is a PqNb structure as well, where $H$ is any 3 -form satisfying

$$
\imath_{P^{\sharp} \alpha \wedge P^{\sharp} \beta} H=0,
$$

for all $\alpha, \beta \in \Omega^{1}(M)$. In particular, when $d B$ satisfies equation (47), then $\left(P, P^{\sharp} B^{b},-d B_{P^{\sharp} B^{b}}, d B\right)$ and $\left(P, P^{\sharp} B^{b},-d B_{P^{\sharp} B^{b}}, 0\right)$ are both PqNb structures. It may also happen that, given a degenerate Poisson bivector $P$ and a $(1,1)$-tensor $A$ such that equation (4) holds, does not exist any $\mathrm{PqNb}$ structure associated with $P$ and $A$ at all. For example, if we take $P$ to be the null bivector and $A$ any non-Nijenhuis tensor, then equation (4) is trivially satisfied but equation (6) can never hold.

\subsection{Compatibility with reduction}

Now we will consider the concepts of gauge transformation and reduction of $\mathrm{PqNb}$ structures and prove that they commute.

Theorem 3.16. Let $(M, P, A, \phi, H)$ be a Poisson quasi-Nijenhuis manifold with background, $i_{N}: N \subset M$ a submanifold and $E$ a vector subbundle of $\left.T M\right|_{N}$ as in Definition 2.1, and suppose that all the conditions of Theorem 2.7 are satisfied, so that $(M, P, A, \phi, H)$ is reducible to a Poisson quasiNijenhuis manifold with background $\left(Q, P^{\prime}, A^{\prime}, \phi^{\prime}, H^{\prime}\right)$. Let also $B$ be a 2form on $M$ such that:

(a) $B^{b}(T N) \subset E^{0}$;

(b) $B$ is projectable to a 2 -form $B^{\prime}$ on $Q$.

Consider the gauge transformation of $(P, A, \phi, H)$ associated with $B,(\tilde{P}, \tilde{A}, \tilde{\phi}, \tilde{H})$, as in Theorem[3.1. Then, $(M, \tilde{P}, \tilde{A}, \tilde{\phi}, \tilde{H})$ reduces to a Poisson quasi-Nijenhuis manifold with background $\left(Q, \tilde{P}^{\prime}, \tilde{A}^{\prime}, \tilde{\phi}^{\prime}, \tilde{H}^{\prime}\right)$ which is also the gauge transformation of $\left(P^{\prime}, A^{\prime}, \phi^{\prime}, H^{\prime}\right)$ associated with $B^{\prime}$. In other words, the diagram

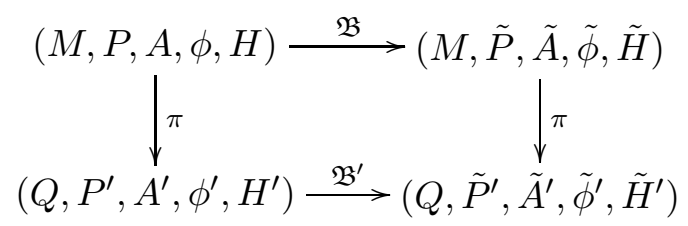

is commutative, where $\mathfrak{B}, \mathfrak{B}^{\prime}$ are the gauge transformations on $\mathfrak{C}_{P q N b}(M)$, $\mathfrak{C}_{P q N b}(Q)$ associated with $B, B^{\prime}$, respectively, and $\pi: N \rightarrow Q$ is the canonical projection. 
Proof. The gauge transformation of $\left(P^{\prime}, A^{\prime}, \phi^{\prime}, H^{\prime}\right)$, associated with $B^{\prime}$, is the PqNb structure $\left(\tilde{P}^{\prime}, \tilde{A}^{\prime}, \tilde{\phi}^{\prime}, \tilde{H}^{\prime}\right)$ on $Q$ where $\tilde{P}^{\prime}=P^{\prime}, \tilde{A}^{\prime}=A^{\prime}+C^{\prime}$, $\tilde{\phi}^{\prime}=\phi^{\prime}-d B_{C^{\prime}}^{\prime}-d\left(\imath_{A^{\prime}} B^{\prime}\right)$, and $\tilde{H}^{\prime}=H^{\prime}+d B^{\prime}$, with $C^{\prime}$ denoting the $(1,1)$ tensor $P^{\prime \sharp} B^{\prime \prime}$. Therefore, by Definition 2.6, we have to prove that the tensors $\tilde{P}, \tilde{A}, \tilde{\phi}, \tilde{H}$, given by equations (31)-(34), project respectively to the tensors $\tilde{P}^{\prime}, \tilde{A}^{\prime}, \tilde{\phi}^{\prime}, \tilde{H}^{\prime}$. By assumption, $P, A, \phi, H, d B$ project to $P^{\prime}, A^{\prime}, \phi^{\prime}, H^{\prime}, d B^{\prime}$, respectively. Moreover, $C$ projects to $C^{\prime}$ (see the proof of Theorem 3.1 in [18]; condition (a) above, as well as condition (ii) in Theorem 2.7, are needed here) and this implies that $B_{C}$ projects to $B_{C^{\prime}}^{\prime}$ :

$$
\begin{aligned}
\left(\pi^{*} B_{C^{\prime}}^{\prime}\right)(X, Y) & =B^{\prime}\left(C^{\prime} \pi_{*} X, \pi_{*} Y\right) \circ \pi=\left(\pi^{*} B^{\prime}\right)\left(\left.C\right|_{T N} X, Y\right) \\
& =\left(i_{N}^{*} B\right)\left(\left.C\right|_{T N} X, Y\right)=\left(i_{N}^{*} B_{C}\right)(X, Y)
\end{aligned}
$$

for all projectable vector fields $X, Y \in \mathfrak{X}^{1}(N)$, so that in particular $d B_{C}$ projects to $d B_{C^{\prime}}^{\prime}$. With a similar reasoning, we prove that $\imath_{A} B$ projects to ${ }^{\imath} A^{\prime} B^{\prime}$ and consequently we have the same for their exterior derivatives.

\section{Appendix}

Proof of Lemma 3.2. For equations (35) and (36) see reference [12], formulas (B.3.9) and (B.3.8), respectively. As for equation (37), we have

$$
\begin{aligned}
& d_{C} B_{C}(X, Y, Z)=(C X) B(C Y, Z)-(C Y) B(C X, Z)+(C Z) B(C X, Y) \\
& \text { - } B(C[C X, Y], Z)-B(C[X, C Y], Z)+B\left(C^{2}[X, Y], Z\right) \\
& +B(C[C X, Z], Y)+B(C[X, C Z], Y)-B\left(C^{2}[X, Z], Y\right) \\
& \text { - } B(C[C Y, Z], X)-B(C[Y, C Z], X)+B\left(C^{2}[Y, Z], X\right) \\
& =d B(C X, C Y, Z)+B([C X, C Y], Z)+d B(C Y, C Z, X)-(C Y) B(C Z, X) \\
& +\quad(C Z) B(C Y, X)+B([C Y, C Z], X)+d B(C Z, C X, Y)+(C X) B(C Z, Y) \\
& +B([C Z, C X], Y)-d B_{C^{2}}(X, Y, Z) \\
& =\mathcal{B}^{C, C}(X, Y, Z)-d B_{C^{2}}(X, Y, Z)-P\left(\left[B^{b} X, B^{b} Y\right]_{P}, B^{b} Z\right) \\
& \text { - } P\left(\left[B^{\mathrm{b}} Y, B^{\mathrm{b}} Z\right]_{P}, B^{\mathrm{b}} X\right)-P\left(\left[B^{\mathrm{b}} Z, B^{\mathrm{b}} X\right]_{P}, B^{\mathrm{b}} Y\right) \\
& +P^{\sharp}\left(B^{b} Y\right) P\left(B^{b} Z, B^{b} X\right)-P^{\sharp}\left(B^{b} Z\right) P\left(B^{b} Y, B^{b} X\right)-P^{\sharp}\left(B^{b} X\right) P\left(B^{b} Z, B^{b} Y\right) \\
& =\mathcal{B}^{C, C}(X, Y, Z)-d B_{C^{2}}(X, Y, Z)+d_{P} P\left(B^{\mathrm{b}} X, B^{b} Y, B^{\mathrm{b}} Z\right) \\
& =\mathcal{B}^{C, C}(X, Y, Z)-d B_{C^{2}}(X, Y, Z) \text {, }
\end{aligned}
$$

for all $X, Y, Z \in \mathfrak{X}^{1}(M)$, where we used the fact of $P$ being Poisson in the third and in the last equalities.

Proof of Lemma 3.3. For proving (40), we take $\alpha \in \Omega^{1}(M)$ and apply it on the right hand side (RHS) of the equation. This gives, using (38), (39) 
and (3),

$$
\begin{aligned}
& \alpha(\mathrm{RHS})=-d B\left(A X, Y, Q^{\sharp} \alpha\right)-d B\left(X, A Y, Q^{\sharp} \alpha\right)+d\left(\imath_{A} B\right)\left(X, Y, Q^{\sharp} \alpha\right) \\
& \quad-H\left(C X, Y, Q^{\sharp} \alpha\right)-H\left(X, C Y, Q^{\sharp} \alpha\right) \\
& \quad=\quad-(A X) B\left(Y, Q^{\sharp} \alpha\right)+B\left([A X, Y], Q^{\sharp} \alpha\right)-B\left(\left[A X, Q^{\sharp} \alpha\right], Y\right) \\
& \quad+\quad(A Y) B\left(X, Q^{\sharp} \alpha\right)+B\left([X, A Y], Q^{\sharp} \alpha\right)+B\left(\left[A Y, Q^{\sharp} \alpha\right], X\right) \\
& \quad+X B\left(Y, A Q^{\sharp} \alpha\right)-Y B\left(X, A Q^{\sharp} \alpha\right)-B\left(A[X, Y], Q^{\sharp} \alpha\right)-B\left([X, Y], A Q^{\sharp} \alpha\right) \\
& \quad+B\left(A\left[X, Q^{\sharp} \alpha\right], Y\right)-B\left(A\left[Y, Q^{\sharp} \alpha\right], X\right)-\mathcal{C}_{Q, A}\left(B^{b} X, \alpha\right)(Y)+\mathcal{C}_{Q, A}\left(B^{b} Y, \alpha\right)(X) \\
& \quad=B\left([A X, Y], Q^{\sharp} \alpha\right)+B\left([X, A Y], Q^{\sharp} \alpha\right)-B\left(A[X, Y], Q^{\sharp} \alpha\right)-B\left([X, Y], A Q^{\sharp} \alpha\right) \\
& \quad-\alpha(A[C X, Y])+\alpha([C X, A Y])+\alpha(A[C Y, X])-\alpha([C Y, A X]) \\
& \quad=\alpha([A X, C Y]-A[C X, Y]-A[X, C Y]+A C[X, Y] \\
& \quad+\quad[C X, A Y]-C[A X, Y]-C[X, A Y]+C A[X, Y]),
\end{aligned}
$$

for any $X, Y \in \mathfrak{X}^{1}(M)$. As for (41), we have

$$
\begin{aligned}
d_{A} & B_{C}(X, Y, Z)+d_{C}\left(\imath_{A} B\right)(X, Y, Z)=(A X) B(C Y, Z)-(A Y) B(C X, Z) \\
& +(A Z) B(C X, Y)+(C X)\left(\imath_{A} B\right)(Y, Z)-(C Y)\left(\imath_{A} B\right)(X, Z) \\
& +(C Z)\left(\imath_{A} B\right)(X, Y)-B\left(C[X, Y]_{A}, Z\right)+B\left(C[X, Z]_{A}, Y\right)-B\left(C[Y, Z]_{A}, X\right) \\
& -\left(\imath_{A} B\right)\left([X, Y]_{C}, Z\right)+\left(\imath_{A} B\right)\left([X, Z]_{C}, Y\right)-\left(\imath_{A} B\right)\left([Y, Z]_{C}, X\right) \\
& =\mathcal{B}^{A, C}(X, Y, Z)+\mathcal{B}^{C, A}(X, Y, Z)-d B_{A C}(X, Y, Z)-d\left(\imath_{C A} B\right)(X, Y, Z) \\
& -C_{Q, A}\left(B^{b} X, B^{b} Y\right)(Z)-C_{Q, A}\left(B^{b} Y, B^{b} Z\right)(X)-C_{Q, A}\left(B^{b} Z, B^{b} X\right)(Y) \\
& =\mathcal{B}^{A, C}(X, Y, Z)+\mathcal{B}^{C, A}(X, Y, Z)-d B_{A C}(X, Y, Z)-d\left(\imath_{C A} B\right)(X, Y, Z) \\
& +\mathcal{H}^{C, C}(X, Y, Z),
\end{aligned}
$$

for all $X, Y, Z \in \mathfrak{X}^{1}(M)$.

Proof of Lemma 3.4. One just has to expand $d_{A}\left(\imath_{A} B\right)$ and then use (42):

$$
\begin{aligned}
d_{A}\left(\imath_{A} B\right)(X, Y, Z)=(A X)\left(\imath_{A} B\right)(Y, Z)-(A Y)\left(\imath_{A} B\right)(X, Z)+(A Z)\left(\imath_{A} B\right)(X, Y) \\
\quad-\left(\imath_{A} B\right)\left([X, Y]_{A}, Z\right)+\left(\imath_{A} B\right)\left([X, Z]_{A}, Y\right)-\left(\imath_{A} B\right)\left([Y, Z]_{A}, X\right) \\
=\mathcal{B}^{A, A}(X, Y, Z)-d B_{A, A}(X, Y, Z)-B^{b}(Z)\left(\mathcal{N}_{A}(X, Y)\right) \\
\quad+B^{b}(Y)\left(\mathcal{N}_{A}(X, Z)\right)-B^{b}(X)\left(\mathcal{N}_{A}(Y, Z)\right) \\
=\mathcal{B}^{A, A}(X, Y, Z)-d B_{A, A}(X, Y, Z) \\
\quad-B^{b}(Z)\left(Q^{\sharp}\left(\imath_{X \wedge Y} \phi+\imath_{A X \wedge Y} H+\imath_{X \wedge A Y} H\right)\right) \\
\quad+B^{b}(Y)\left(Q^{\sharp}\left(\imath_{X \wedge Z} \phi+\imath_{A X \wedge Z} H+\imath_{X \wedge A Z} H\right)\right) \\
\quad-B^{b}(X)\left(Q^{\sharp}\left(\imath_{Y \wedge Z} \phi+\imath_{A Y \wedge Z} H+\imath_{Y \wedge A Z} H\right)\right) \\
=\quad \mathcal{B}^{A, A}(X, Y, Z)-d B_{A, A}(X, Y, Z)+\left(\imath_{C} \phi\right)(X, Y, Z) \\
\quad+\mathcal{H}^{A, C}(X, Y, Z)+\mathcal{H}^{C, A}(X, Y, Z),
\end{aligned}
$$

for all $X, Y, Z \in \mathfrak{X}^{1}(M)$. 


\section{Acknowledgements}

This work has been partially supported by FCT grant SFRH/BD/44594/2008

(FC) and by CMUC-FCT and project PTDC/MAT/69635/2006 (JMNdC).

\section{References}

[1] Antunes P., Poisson quasi-Nijenhuis structures with background, Lett. Math. Phys., 86 (2008) 33-45.

[2] Bursztyn H. and Radko O., Gauge equivalence of Dirac structures and symplectic groupoids, Ann. Inst. Fourier, 53 (1) (2003) 309-337.

[3] Bursztyn H., Cavalcanti G.R. and Gualtieri M., Reduction of Courant algebroids and generalized complex structures, Adv. Math., 211 (2) (2007) 726-765.

[4] Caseiro R., De Nicola A. and Nunes da Costa J.M., On Poisson quasiNijenhuis Lie algebroids, arXiv:0806.2467 v1 [math.DG].

[5] Crainic M., Generalized complex structures and Lie brackets, arXiv:math/0412097v2 [math.DG].

[6] Falceto F. and Zambon M., An extension of the Marsden-Ratiu reduction for Poisson manifolds, Lett. Math. Phys., 85 (2008) 203-219.

[7] Gualtieri M., Generalized Complex Geometry, arXiv:math/0401221v1 [math.DG].

[8] Hitchin N., Generalized Calabi-Yau manifolds, Quart. Journal Math., 54 (3) (2003) 281-308.

[9] Hu S., Hamiltonian symmetries and reduction in generalized geometry, Houston J. Math., 35 (3) (2009) 787-811.

[10] Lin Y. and Tolman S., Symmetries in generalized Kähler geometry, Comm. Math. Phys., 268 (1) (2006) 199-222.

[11] Lindström U., Minasian R., Tomasiello A. and Zabzine M., Generalized complex manifolds and supersymmetry, Comm. Math. Phys., 257 (1) (2005) 235-256.

[12] Magri F. and Morosi C., A geometrical characterization of integrable Hamiltonian systems through the theory of Poisson-Nijenhuis manifolds, Quaderno S 19, Univ. of Milan, 1984.

[13] Marsden J. and Ratiu T., Reduction of Poisson Manifolds, Lett. Math. Phys., 11 (1986) 161-169. 
[14] Ševera P. and Weinstein A., Poisson geometry with a 3-form background, Prog. Theor. Phys. Suppl., no 144 (2001) 145-154.

[15] Stiénon M. and Xu P., Poisson Quasi-Nijenhuis Manifolds, Comm. Math. Phys., 270 (2007) 709-725.

[16] Stiénon M. and Xu P., Reduction of generalized complex structures, J. Geom. Phys., 58 (2008) 105-121.

[17] Vaisman I., Complementary 2-forms of Poisson structures, Compositio Mathematica, 101 (1) (1996) 55-75.

[18] Vaisman I., Reduction of Poisson-Nijenhuis manifolds, J. Geom. Phys., 19 (1996) 90-98.

[19] Vaisman I., Reduction and submanifolds of generalized complex manifolds, Differential Geom. Appl., 25 (2007) 147-166.

[20] Zambon M., Reduction of branes in generalized complex geometry, J. Symplectic Geom., 6 (4) (2008) 353-378.

[21] Zucchini R., The Hitchin model, Poisson-quasi-Nijenhuis geometry and symmetry reduction, J. High Energy Phys., no 10 (2007) 075. 